\title{
MARIANO AZUELA: \\ PRECURSOR DE LOS NUEVOS NOVELISTAS
}

\author{
POR \\ LUIS LEAL \\ University of California, Santa Bárbara
}

Las contribuciones de Mariano Azucla (1873-1952) al desarrol 10 de 1 a novela mexicana, y cn cicrtos aspectos a la hispanoamcricana, no se limitan a una ćpoca o a una forma. La suya es una narrativa en movimiento, en constante búsqueda de la cstructura apropiada para dar expresión a los temas de la hora. Durante el porfiriato escribe sobre temas candentes entonces, como lo fucron los conflictos entre conscrvadores y liberales, entre hacendados y campesinos, entre pobres y ricos. Para cllo utiliza las técnicas y formas de los naturalistas curopeos, de las cuales se apropió para modelar el material nacional. Durante 1a Revolución forja nuevas formas y adapta cl discurso a las circunstancias; cuando los vanguardistas introducen una nueva estética, Azucla la asimi la y publica novelas de acucrdo con csa modalidad; durante los años de reajuste político y social posrevolucionario, vuclve a escribir novelas de denuncia, mas sin repetirse. Si antes escribía sobre 1 as injusticias en las árcas rurales, ahora enfoca los defectos urbanos.

Azucla cs, al mismo tiempo, el primer novelista que ve con claridad 1a malversión de los ideales revolucionarios, y el primero en criticar a los caudil los que no cumplían con los principios por los cuales peleaban. Esa conciencia del fracaso de la Revolución es lo que da forma a las novelas que escribe a partir de 1911. En su obra encontramos un espíritu de inconformidad y rebeldía y un ardiente desco de luchar por mejorar las condiciones de vida del pucblo mexicano.

Si bien conscientes de otros valores presentes en las novelas de Azucla, entre cllos el social, en esta breve apreciación de su obra nos ocuparemos solamente de 1 as relaciones entre la historia y la novela como género literario. Los interesados en otros aspectos de su narrativa, no menos importantes, pueden consultar, entre otros, los cstudios de Gerhard R. Herbst, Eliud Martínez, Stanley L. Robe, Arturo Rivas Sáinz y Jorge Ruffincll i, cnumcrados en la bibliografía final de este csludio.

Las novelas de Mariano Azucla pueden ser clasificadas como representantes de cuatro tendencias narrativas bien definidas: las naturalistas, publicadas o escritas antes de 1910; 1as de la Revolución, que aparecen entre 1911 y 1918; 1as 
vanguardistas (1923-1932) y ías políticas, que comienza a escribir en 1937 y conlinúa haciéndolo hasta cl año de su muerte.

Como novelista, Azuela inicia su carrera bajo la influencia de los realistas españoles y los naturalistas francescs. En cl ensayo sobre José Joaquín Fernández de Lizardi recogido en Cien años de novela mexicana justifica cl poco aprecio que tenia por El Periquillo Sarniento diciendo que encuentra "plausible cl interés vivo que algunos letrados reclaman para esta obra y explicable el aburrimicnto que nos dio a los que nos cducamos con la novela del siglo XIX, con las obras de un Balzac, de un Zola, de Maupassant o con 1as de Pereda, Valera y Pérez Galdós (Obras completas III, 584)'. ¿Por qué abandonó Azucla cl naturalismo, que tanto admiraba? Tal ve bajo la influcncia de fucrzas sociales, estoes, los acontecimicntos ocurridos cntre 1910 y 1920 que cambiaron 1a historia del pais y su fe en la revolución

Para cl autor de Los de abajo la novela mexicana anterior a la Revolución no había logrado producir obras a la altura de las curopeas. Su crítica de la novela nacional 1 a inicia preguntando ¿Hay novcla mexicana? y aunque no contesta su propia pregunta, sí dice: "Por mi parte confieso que sólo por necesidad de mi oficio me pusc a 1 ecr 1 a mayor parte de las novelas [mexicanas] del siglo XIX, y después de cumplir con esta fastidiosa tarea, me di cuenta de que sólo por comparación de nucstros propios autores, merccen los clogios calurosos los mejores" (III, 572 ). Si bien no lo dice, podemos deducir que consideraba sus propias novelas como 1 as primeras en México que podían equipararse a las de otros paises. Y en verdad, fueron las novclas de Azuela las primeras en ser traducidas a otros idiomas y en dar a conocer la narrativa mexicana en el exiranjero.

Comocrítico, Azucla es muy severo con sus precursores. A Federico Gamboa, a quien consideraba como "figura de primer orden en la narrativa mexicana", ic critica "su afán de sacar moral de todo, por procedimientos impropios al arte"; lo que indica, por supuesto, que todo es posible en la novela mientras el autor no pierda de vista el valor estético de la obra. Lo anterior lo decía Azuela en 1947 (fecha de la conferencia que dictó en el Colegio Nacional en la cual habló sobre Gamboa) y tal vez 1 os elementos artísticos en 1a novela no 1e preocupaban tanto durante los años cuando escribió sus primeras narraciones, la ćpoca de María Luisa (1907), Los fracasados (1908), Mala yerba (1909) y Sin amor (publicada cn 1912 pero escrita antes), obras no muy diferentes de las de los naturalistas mexicanos a quienes tanto criticaba. Si Azucla hubicra dejado de publicar en 1910, hoy sería

\footnotetext{
${ }^{1}$ En adelante nos referiremos a 1 as Obras completas de Azuela indicando solamente el volumen y la página.
} 
considerado como un novelista menor. Sin embargo, a partir de ese año abandona cl naturalismo y crea una nueva novela, divorciada de la europea y precursora de 1a que hoy consideramos como la auténtica novela hispanoamericana.

¿Cómo ocurrió esa transformación? Sin duda fue debida al cambio de ideas que sufrió Azuela como resultado de 1 os acontecimientos políticos ocurridos en 1911. La Revolución maderista había sido subvertida. "No se hacían 1as clecciones generales todavía, cuando el gobicrno provisional del presidente don Francisco Lcón de 1a Barra [1911] se habia convertido en madriguera de tejones que por medio de combinaciones $\mathrm{c}$ intrigas políticas estaban socavando profundamente los cimicntos del nucvo régimen" (III, 1070). Azucla ese mismo año renuncia a su puesto de jefe político đe Lagos de Moreno (su cantón natal) y de al 1 í cn adclante se dedica a delatar a los "tcjones" en sus novelas". "Desde entonces dejé de ser [...] cl obscrvador screno e imparcial que me había propuesto $\mathrm{en}$ mis cuatro primeras novelas. Ora como testigo, ora como actor en los sucesos que sucesivamente me servirían de base para mis escritos, tuve que ser y lo fui de hecho, un narrador parcial y apasionado [...]. El conflicto íntimo que se me presentó está ficlmente traducido en uno de los diálogos de mi pequeña novela, Andrés Pérez, maderista [1911]" (III, 1070).

Lo que no nos dice Azucla es que en esa novela también abandona las técnicas narrativas finiscculares que había utilizado anteriormente, lo mismo que 1 as formas que prevalecían en la novcla hispanoamericana en general. Si en Andrés Pérez, maderista victte todo su desencanto político, también crea con ella un nucvo discurso narrativo, discurso que scrá adaptado por ios novelistas de la Revolución; discurso en el cual desaparece cl costumbrismo, en el que predomina cl diálogo sobre las largas descripciones del ambiente, en donde se caracteriza a los personajes por medio de las acciones y no de los recursos tradicionales, esto es, el retrato físico o psicológico desde la perspectiva del autor omnisciente. Y lo más importante, por primera vez cl protagonista Andrés Pérez, que es a la vez el narrador a través de cuya conciencia cl lector ve el mundo de la novela, deja de ser heroico para convertirse en un antihéroc, un personaje que ya no es ni bueno ni malo, sino que obra de acuerdo con las circunstancias que se le presentan en la vida diaria.

No todo, por supuesto, es novedoso en Andrés Pérez, maderista. Los personajes, con pocas excepciones, siguen siendo cstereotipos: cl periodista (Don Cuco), el cacique (cl coronel Hernández), cl rebelde (Vicente). Dos de cllos, Andrés Pérez y Toño Reyes, sin cmbargo, trascienden 1a caracterización este-

${ }^{2}$ Para otro punto de vista consúltese el libro de Ruffinelli. 
reotipada. Esto lo consigue Azuela, como ya dijimos, presentando al personaje como posecdor de un carácter ambiguo, recurso ya observado por Carlos Fuentes y scgún el cual los novelistas de la Revolución, "por encima de sus posibles defectos técnicos y a pesar de su lastre documental, introducen una nota original en la novela hispanoamericana: introducen 1a ambigücdad. Porque en la dinámica revolucionaria los héroes pueden ser vill lanos y los vil lanos pueden ser hérocs"(15).

Al mismo tiempo, en Andrés Pérez, maderista Azucla introduce temas sociales y políticos antes no tratados en la narrativa mexicana. Es verdad que ya cn sus novelas naturalistas había criticado a los hacendados, al clero y, veladamente, al gobicrno porfirista. En Andrés Pérez, en cambio, 1a crílica del gobicrno, de los políticos (los "tcjones"), de los periodistas, de los caudillos, es lo que da forma a la narrración. "Me regocijo - dice uno de los personajes - de que la intelectualidad de mañana lave el borrón afrentoso de este Gobierno apático, caduco y servil; de que dé esperanzas de ser menos miscrable, menos venal y menos canal la que la de hoy" (II, 765). Ni el maderismo, al que tanto defendió, se le cscapa en esta primera novela de la Revolución: "Quise condenar, en menos de un centenar de páginas, un aspecto del movimiento de Madero, cuyo triunfo rápido fue la causa de su caida" (III, 1072). Ese discurso crítico es lo que encontramos en toda su obra postcrior a 1911, con excepción de aquell as obras que sospechamos fucron escritas años antes de ser publicadas, como ocurre con Los caciques y Sin amor.

Por primera vez en su obra, Azucla hace uso en Andrés Pérez del humor y 1a sálira para ridiculizar 1 as acciones de los "tejones". Irónicamente, Andrés Pérez, cl narrador protagonista, periodista incpto, $11 \mathrm{cga}$ a ser caudillo del maderismo. Pero el aspecto cómico en la obra nunca $11 \mathrm{cga}$ a sobreponerse sobre la actitud pesimista; la mucrte de Toño Reyes, el idealista, es inútil; los más rabiosos enemigos de Madero han sabido climinarlo para recobrar el poder. La muerte de Reyes es simbólica de 1a muerte del maderismo. En este sentido la obrita es profética, ya que dos años más tarde Madero había de ser asesinado. "Cuantos tuvimos los ojos abicrtos, esperábamos de un momento a otro el derrumbamicnto del régimen maderista, pero no el atentado brutal de que fue víctima" (III, 1072). Esto confirma la tcoría de Agustín Yáñez, para quien la característica principal de la novela mexicana es su elemento profético?.

3 Véase nuestro estudio, "Agustín Yánez y la novela mexicana. Rescate de una teoría". Revista lberoamericana 48,118-119 (1982): 121-129. 
La novcla corta Los caciques: Del Llano Hermanos, S. en C., terminada en plena Revolución, esto es, en junio de 1914 (precisamente cuando triunfa el vil 1 ismo con 1a toma de Zacatecas) pero no publicada hasta 1917, es comoAndrés Pérez, maderista, obra de transición. El tema no es la Revolución, sino cl conflicto - a la mancra naturalista - entre pobres y ricos en un pucblo del interior. Si bien el subtítulo cs "Novela de 1a Revolución Mexicana" y la acción se desarrolla entre 1912 y 1913, 1a Revolución es sólo tema de conversación o de discurso político. En cl último corto capítulo (menos de dos páginas) en donde se dramatiza la lucha armada, esto es, la toma del pucblo, la escena nos da la impresión de scr un simple cjercicio en el arte de narrar. La acción no está vista, como otra escena semejante en Los de abajo, desde la perspectiva de los revolucionarios, sino de los habitantes del pucblo ("Las gentes corrían y se atropcllaban. '¡Ya están aquí!' ‘YYa cstán aquí!'), o del autor omnisciente ("Las puerlas se cerraban con estruendo y las calles quedaban solas. Sc oyó, primero, un disparo [...] apareció al galope un grueso grupo de montados, con los fusiles a la cara [...] comenzó el saqueo [...]" [II, 865]).

Si no fuera por cste último capítulo, en cl cual Azucla usa la Revolución para terminar con un descnlace dramático, Los caciques muy bien podría ser considerada como novela de la primera ćpoca. Tal vez la mayor parte de el la fue escrita antes de que se inciara cl conflicto, y la terminó después del triunfo. Pero cuando aparece, ya Azucla había publicado Los de abajo (novicmbre, 1915), novela revolucionaria tanto en el contenido como $\mathrm{en} \mathrm{la} \mathrm{estructura.} \mathrm{¿Cómo} \mathrm{se} \mathrm{explica} \mathrm{el}$ cambio? He aqui una conjetura. Cuatro meses después de haber terminado de escribir Los caciques, Azucla se unc a los villistas. ("En los últimos días de octubre de 1914 me incorporć al Estado Mayor de Julián Medina, en Irapuato" [III, 1079]). Es en cl cjército revolucionario, en su capacidad de médico militar, que Azucla comienza a escribir Los de abajo, utilizando las anécdotas que le contaba Medina. En Tepatillán, Jalisco, conoce a otro revolucionario villista, Manuel Caloca, quien Ic sirve como modelo para el protagonista de la novela en cicrne. Como los carrancistas (los federales en la novela) no les daban tregua, Azucla y Caloca, quien iba herido, abandonan Tepatilán y se refugian en el cañón de Juchipila. "Con Caloca en angarillas, una partida de carrancistas nos sorprendió en el fondo del cañón, pero como la gente del coronel [Caloca] cra de serranos y caballistas magníficos, con facilidad se apoderaron de las alturas y pronto pusicron en fuga al cnemigo. Yo, cntretanto, al amparo de un covachón abicrto en la peña viva, tomaba apuntes para la escena final de la novcla apenas comenzada" (III, 1087). La obra, como es bien sabido, la terminó Azucla en El Paso, Texas, cn donde la publicó cn el folletín del periódico El Paso del Norte entre octubre y noviembre de $1915^{4}$.

${ }^{4}$ Para la historia de la edición periodística de Los de abajo consúltese el libro de Robe. 
Lo que nos interesa subrayar aquí es lo siguiente. Si bien es verdad que de acuerdo con el credo naturalista el narrador debía de pintar el ambiente y los personajes con objetividad, en Azucla - y en general en casi todos los naturalistas hispanoamericanos- predomina más la influencia de las novelas naturalistas francesas que 1a realidad circundante. Aunque Guillermo Ara, en su estudio sobre la novela naturalista hispanoamericana, observa que las tres primeras obras de Azuela "cumplen en cierto modo una aproximación măs certera que la de Gamboa al método naturalista"(59) los términos que usa para analizarlas no lo indican. María Luisa le recuerda a Irresponsable de Podestá porque Azuela en esa primera novela "maneja sus muñecos con cicrto desparpajo cínico" (59). Si los personajes son "muñecos" y si están captados con "cinismo", la actitud naturalista hacia la realidad desaparece. El mismo Azucla confesó que en Sin amor se propuso dar "un boceto del ambiente de sopor y aburrimicnto de las poblaciones cortas, que tan magistralmente supo describir Santiago Rusiñol en El pueblo gris" (III, 1064).

En Los de abajo, en cambio, Azuela se olvida de la novela curopea (no sabemos si conocía a Podestá) para forjar su propia novcla, con su propia cstructura y su propio acercamiento a la realidad. Por primera vez capta directamente la vida y ia naturaleza que le rodea, sin tratar de ajustarlas a modelos narrativos preconcebidos. Para estructurar la narración hace uso de lo que 1e cuentan los oficiales, los soldados rasos y las soldaderas, lo mismo que la gente que encuentra en los pucblos y las comunidades rurales. Los personajes no son muñecos, son los mismos revolucionarios; las acciones las que observó durante un año en cl campo de batalla, en largas caminatas, en pucblos destruidos o en viajes por ferrocarril donde las conversaciones giran en torno al tema "yo maté". La novela se abre con el triunfo del guerrillero Demetrio Macías en el Cañón de Juchipila y termina allí mismo con su mucrte. Esta estructura circular da a la novela cierta armonía consonante con la naturaleza de los hechos relatados. Que Azucla conscientemente haya dado preferencia a csta forma artística - cuando comienza a cscribir la novela ya sabe cómo va a terminar-es un rasgo genial que le coloca por encima de la mayor parte de los novelistas de la Revolución, simples relatores del devenir histórico. En Los de abajo Azuela abandona por completo las normas de los novclistas curopcos y forja una nueva forma narrativa, genuinamente americana, admirablemente adaptada al tema. Su creación ha de tener resonancias en el desarrollo de la novela hispanoamericana.

Para que Azucla introduzca otras innovaciones en cl arte de narrar hay que esperar hasta la tercera época cn su desarrollo como novelista, la llamada ctapa vanguardista, ya que sus otras novelas de la Revolución (publicadas en 1918) no son superiores a Los de abajos. No importa cl motivo por cl cual Azucla decidió 
utilizar el discurso vanguardista; el hecho es que escribió y publicó tres novelas experimentales, La Malhora (1923), El desquile (1925) y La luciérnaga (1932)6.

La nueva modalidad que encontramos en La Malhora ya nos indroduce a la novela hispanoamericana postmoderna; su estructura es, para la ćpoca, sumamente novedosa; tanto, que fue rechazada por un jurado, al que no impresionó. Hoy, por supuesto, nos 11 ama la atención su estructura: los tiempos superpucstos; la arbitraricdad en cl desarrollo de la anćcdota; los bruscos cambios tcmáticos; los flash backs; la interrupción de las escenas a medio desarrollo; la caracterización parcial de los personajes. Lo más desconcertante es el desarrollo de la trama - cn relación a sus obras anteriores-, ya que no es cronológico. La novela se abre con el asesinato del padre de Altagracia (La Malhora), pero no es hasta cl último cpisodio cuando cl lector descubre que cl asesino, Marcelo, cs también quien deshonró a la hija cuando cra una chiquil la. Además, se presentan cuatro ambientes distintos, unidos solamente a través de la presencia del personaje titular. La acción se inicia y termina en el mismo lugar. Sin cmbargo, la cstructura no es, como en Los de abajo, circular, sino más bien cuadriforme: cuatro ctapas de la vida de una prostituta. La Malhora ya apunta hacia la novela de nuestros días.

La novela mexicana retrazó su desarrollo al no haber premiado csta novela, al no haber cultivado su nueva modalidad. Y cl mismo autor, disgustado con la crítica, abandonó la narrativa experimental para volver a escribir novelas de estructuras y contenidos tradicionales. Durante su última época, con sus novelas de crítica social, no logró superarse ni abrir nucvos derroteros a la narrativa hispanoamericana. De sus últimas novclas sólo en Nueva burguesía encontramos novedades. Aparte de 1a crítica que allí hace del gobierno, en la forma logra integrar los materialcs con más arte. Nos 11 ama 1a atención 1a rapidez con que pasa de escena a escena, sin prcocuparse por unir los diversos episodios. La técnica cs analítica y descriptiva, predominando cl diálogo. La inclinación naturalisła cs evidente en la pintura de los aspectos sórdidos de la vida capitalina. Pero, apartándose de su antcrior apego al naturalismo, cvita las largas y aburridas descripcioncs del ambiente y todo costumbrismo.

Tal vez en su última época Azuela quiso poner en práctica su concepto de la novcla: libro destinado al gran público. Sin cmbargo, tcnía una noción más compleja de lo que debe de ser el género, además del éxito de libreria. La novela, nos dice, debe de proporcionar una cmoción estética. Es por eso por lo cual rechaza

\footnotetext{
${ }^{5}$ Para una evaluación de estas novelas consúltese nuestro libro sobre Azuela.

5 Sobre cstas obras consúltese el libro de Eliud Martínez.
} 
la narrativa mexicana de José Tomás de Cuéll ar y sus precursores. ("La emoción estética que el lector moderno exige de la novela, no la supo dar Facundo, así como tampoco sus precursores" [III, 611]). La novela también, según Azucla, debe cvitar cl convertirse en propaganda o apostolado. Irónicamente, lo que dice de Paul Bourget y Emile Zola, csto es, que "perdicron toda su fuerza en cuanto sc presentaron como reformadores sociales" (III, 611), podria aplicarse a las novelas de su última ćpoca.

La novela tampoco debe de ser, repite Azucla con insistencia, didáctica. El éxito de una novela depende de si es o no es entretenida. "El valor de un drama, de una comedia o de una novela - nos dice-, depende en buena parte de su factura litcraria, pero si su construcción no es sólida, si falta contextura y vida a sus personajes, verosimilitud al medio y acción, interés humano al tema y a los problemas que suscita, la obra es un fracaso" (III, 623).

Personajes que viven, vcrosimilitud ambiental y de acción, interés humano del tema, son precisamente las cualidades que encontramos en las más logradas novclas de Azucla. Su interés en los cambios sociales que ocurrían al momento de escribirlas es lo que resalta en sus obras. La voz de Azucla no es oída en esos momentos, tal vez por el estruendo de los cañones. Ha de pasar más de una década antes de que la mecha de la novela de la Revolución iniciada por Azucla prenda fuego. El águila y la serpiente y La sombra del caudillo de Martín Luis Guzmán aparccen en España 1928 y 1929. Pcro no es hasta despućs de èsos años cuando la Revolución, como tema, llega a predominar en la novela mexicana. Las mejores obras de Gregorio López y Fucntes, Rafacl Muñoz, José Rubén Romero y Cipriano Campos Alatorre se publican entre 1930 y 1940.

El legado de Azucla no termina alli, con 1os narradores de la Revolución. Su presencia la intuimos en Agustín Yáñez, en Juan Rulfo, en Carlos Fuentes, y cn verdad, en algunos de los más grandes narradores hispanoamericanos. A pesar de los defectos que algunos críticos les encuentran, las novelas de Mariano Azucla ayudaron a crear no sólo una conciencia nacional, sino a preparar el terreno para 1a nueva novela hispanoamcricana. 


\section{OBRAS CITADAS}

Ara, Guillermo. La novela naturalista hispanoamericana. Bucnos Aires: EUDEBA, 1965.

Azucla, Mariano. Andrés Pérez, maderista. México: Imprenta de Blanco y Botas, 1911. Recogido en Obras completas, II, 764- 800.

- Cien años de novela mexicana. México: Ediciones Botas, 1947. Recogido en Obras completas, III, 569-668.

- Obras completas. 3 vols. México: Fondo de Cultura Económica, 1958, $1958,1960$.

Fuentes, Carlos. La nueva novela hispanoamericana. México: Editorial Joaquín Mortiz, 1969.

Herbst, Gerhard R. Mexican Society as Seen by Mariano Azuela. New York: Edicioncs Abra, 1977.

Lcal, Luis. Mariano Azuela, vida y obra. México: Ediciones De Andrea, 1961.

Marlincz, Eliud. The Art of Mariano Azuela: Modernism in "La Malhora," "El Desquite." "La Luciernaga." Pitsburg, Pennsylvania: Latin American Litcrary Review Press, 1980.

Robe, Stanley L. Azuela and the Mexican Underdogs. Berkeley: University of California Press, 1979.

Rivas Sáinz, Arturo. El estilo de Mariano Azuela. Guadalajara, Jalisco: Ediciones del Departamento de Bellas Artes, 1974.

Ruffinclli, Jorge. Literatura e ideología: el primer Mariano Azuela (1896-1918). México: Premia Editora de Libros, 1982. 
